\title{
The aging anesthesiologist: Are we asking the right question?
}

\author{
Robert Byrick, MD
}

Received: 28 May 2014/ Accepted: 19 June 2014/Published online: 23 July 2014

(c) Canadian Anesthesiologists' Society 2014

Kahneman, ${ }^{1}$ a Nobel laureate, recognized that we all have intuitive feelings and opinions about almost everything and it is our natural inclination to believe that we are rarely totally "stumped". When a satisfactory answer to a complex question is not readily available, a common intuitive human response is to find a related question that is easier to answer. In this issue of the Journal, Baxter et al. ${ }^{2}$ address an important topic that relates to the professionalism of anesthesiologists in dealing with age-related decline in clinical performance. The authors offer a "proactive" strategy that hospital departments and practice groups should consider for managing this issue. They identify that many anesthesiologists wish to continue in practice beyond age 65 and that the ability of physicians to self-assess has been found to be inadequate to protect patient safety. We have to ask whether this lack of ability to self-assess is limited to this age group (there is abundant evidence that this shortcoming is not age-dependent) and whether the proposed strategy would be expected to improve overall patient safety.

The rationale for undertaking Baxter's review was partly related to the findings by Tessler et al. ${ }^{3}$ that aging anesthesiologists (i.e., older than $65 \mathrm{yr}$ ) have 1.5 times the risk (after adjusting for exposure) of being found responsible in litigation when compared with their younger colleagues. Importantly, the degree of injury, as defined by the financial awards associated with the litigated cases, was greater in patients of the older anesthesiologists. Tessler ${ }^{3}$ acknowledged that his methodology, and hence his conclusions, had limitations due to the low frequency of

R. Byrick, MD ( $ه)$

Department of Anesthesia, St. Michael's Hospital, University of Toronto, 30 Bond Street, Toronto, ON M5B 1W8, Canada

e-mail: robert.byrick@utoronto.ca litigation in clinical practice, the unknown work environment of the different groups, and the unknown underlying mechanism for increased risk. He speculated that the increased risk among older anesthesiologists could have been related to easier fatigability, resulting in reduced vigilance, longer response times, less continuing education, or poorer communication skills. Eger $^{4}$ commented that another confounding factor may have been that the two groups (i.e., older than 65 and younger than 65) were fundamentally different in their training and skill set right from the outset of their professional careers. When dealing with sensitive social and legal issues, we all agree that we must be careful not to draw possibly erroneous conclusions about individuals based on statistical analysis of groups.

There are, however, other data that support Tessler's conclusion that the age of anesthesiologists is a contributing factor to a decline in favourable outcomes in clinical practice. For example, Campbell et al. ${ }^{5}$ reported that $52 \%$ of patient complaints to the General Medical Council in the United Kingdom (UK) involved anesthesiologists qualified for more than 20 years, whereas only $39 \%$ of the cohort were over the age of 45 . Baxter et $a l^{2}$ have asked an important, yet complex, question that requires serious analysis. We cannot, and should not, deny that there may be an unrecognized problem pertaining to some anesthesiologists that is related to an age-related decline in clinical abilities. Nevertheless, in order to develop a strategic approach to enhance patient safety, we need to identify the nature, extent, and consequences of the problem as well as the solution.

The authors ${ }^{2}$ review physiologic changes that occur with aging and speculate that the cause of the increased risk to patient safety may be perceptual limitations (e.g., hearing or visual deficiencies) or cognitive decline, which would influence judgment and decision-making. They propose 
that the "available evidence" suggests a "proactive approach" that could lead to a change in the "default position of continuing practice until a problem is recognized". They propose that the relevant departments and practice groups require their aging colleagues to undergo an annual review, including a demonstration of competence using simulation, to ensure their continued work in the hospital setting. Although this type of proactive approach may be intuitively appealing, the authors recognize that data are lacking to suggest that only older anesthesiologists have these deficiencies or that a simulation-based assessment would be an effective screening tool specifically for aging anesthesiologists.

The authors also suggest that aging anesthesiologists may continue to practice due to a lack of retirement savings. Interestingly, our professional society recognized this issue many decades ago, and senior members of the profession initiated the Canadian Anaesthetists' Mutual Accumulating Fund $^{6}$ to assist in providing financial security. If financial considerations are the underlying cause for anesthesiologists to continue practicing, perhaps inappropriately, our profession should once again examine pensions or further retirement strategies.

Before adopting the strategy suggested by Baxter et al., ${ }^{2}$ we should ask whether their proposal reflects the correct question (i.e., a concern for overall patient safety) or whether it reflects an easier question (i.e., competence of aging anesthesiologists working in hospitals), as Kahneman ${ }^{1}$ suggested was our natural inclination. It should be emphasized that all studies show that the absolute number of adverse events (attributed to anesthesiologists) that lead to complaints or litigation are greater in younger anesthesiologists, although the frequency is relatively higher in those older than 65 .

The suggested proactive strategy ${ }^{2}$ is a hospital-based intervention that may help to reassure the public that patient safety is optimized in hospitals. Nevertheless, one predictable outcome is that aging anesthesiologists who wish to continue in practice might elect to leave hospitals and practice in isolated "out-of-hospital" environments. Out-of-hospital practice often lacks the safety of daily collegial interactions that characterize group practice and supportive advice on patient care decisions. This environment may also limit opportunities for continuing professional development. I would suggest that the practice environment for anesthesiologists of all ages is an important factor in the genesis of adverse events. This may have been another confounding variable in Tessler's study, ${ }^{3}$ as more elderly anesthesiologists may have been working in isolated high-risk environments. Before assuming that the proposed strategy will enhance overall patient safety, we should consider the potential societal impact. This may protect the hospitals (and practice groups) from some medicolegal risk, but overall patient safety from anesthetic complications may not be improved. I would suggest that anesthetic safety is determined at any age by the competence of the provider within the environment in which he/she is practicing. The problem of an aging workforce in anesthesiology is a systemic professional problem that requires a systemic professional approach.

Warner $^{7}$ also considered the environment in which aging anesthesiologists work to be an important factor and proposed a staged change in workload to reduce the impact of fatigue. This staged change included a reduction in night on-call shifts as well as a restriction in the scope of practice and hours worked - both intended as a means of providing an environment that fosters optimal patient care. Where are the data suggesting that the adverse events, which occur specifically in aging anesthesiologists, take place disproportionately at night or after long shifts? Although, as already mentioned, this view is intuitively appealing. Another feature of the operating room environment today is the increasing presence of anesthesia assistants acting as part of the anesthesia care team and providing important support in managing difficult clinical problems. Optimizing the operating room environment may allow anesthesiologists of all ages to provide safer patient care without "taking away the keys". 7 If the fundamental problem is the inability of anesthesiologists to self-assess and match their competencies to patient care needs in the environment in which they work, then addressing the environment and their self-assessment capabilities are key to improving safety for anesthesiologists of all ages.

Kahneman ${ }^{1}$ also analyzed decision-making in disciplines such as medicine. He pointed out that the acquisition of expertise in complex tasks takes time "because expertise in a domain is not a single skill, but rather a large collection of miniskills". He suggested that the same professional may be highly expert in some tasks in his/her domain, while remaining a novice in others. This is the fundamental problem with using simulation-based assessments alone as a measure of global competence - it cannot assess the full range of "miniskills" required of a practicing anesthesiologist and has never been validated to do so. Warner ${ }^{7}$ asserts that many older physicians compensate well for their lack of "up to date" knowledge with their experience. Kahneman ${ }^{1}$ concluded that the likelihood for professionals to develop intuitive expertise, which characterizes experienced clinical decision-making, depends on the quality and speed of feedback in the clinical setting as well as on sufficient opportunity to practice. He specifically mentioned that anesthesiologists benefit from feedback in clinical practice because the effects of their actions are likely to be immediately evident. He proposes that anesthesiologists are in a better position than many other specialists to 
develop useful intuitive skills, specifically as a result of the feedback inherent in their work. This assumes that they take advantage of these opportunities for feedback or practice in a simulated environment. The value of simulation for anesthesiologists of all ages may not be only in practicing technical and non-technical skills or in managing unpredictable rare clinical crises in a controlled environment. A largely unrecognized benefit of simulation is affording the clinical anesthesiologist the opportunity to practice self-reflection and to receive objective feedback on performance from peers. If the rationale for the adoption of a "proactive" strategy to address the problems of aging anesthesiologists is their lack of ability to self-assess these skills, then arguably, this is the problem that should be addressed. Kahneman ${ }^{1}$ stressed that professionals in many areas of practice have not "learned to identify the situations and the tasks in which their intuition will betray them", and these unrecognized limits of professional skill help to explain why experts (of all ages) are often overconfident.

We must be careful not to deny that problems related to aging exist in the anesthesia workforce - but we must understand and develop strategies to address the precise nature and extent of the problem. Tessler ${ }^{3}$ was correct more work is needed. It is somewhat ironic that our specialty in Canada is moving toward competence-based assessment of residents, ${ }^{8}$ yet Baxter et al. ${ }^{2}$ focus on an agebased (time-based) model for continuing professional competence. The idea may be intuitively appealing, but is it the answer to the correct question with respect to patient safety? In a similar situation, the appropriate relationship between surgical resident work hours and adverse events was intuitively assumed, and shifts were reduced in the name of patient safety. Nevertheless, recent reviews of this experience have documented that the expected improvement in patient outcomes has not been confirmed - in fact, outcomes may be worse! $!^{9}$ This simply emphasizes that we need to be aware of unexpected consequences of decisions based on assumptions that we believe are true but may be founded on unknown preconceived notions.

Another option to consider as a "proactive" approach to the problem would be to ensure that all anesthesiologists have purposeful periodic assessments of their patient outcomes throughout their career - not as a means to retain hospital privileges, but as a professional responsibility and a guideline to practice. This would not entail a single simulation-based high-risk assessment at age 65 , but rather, a meaningful peer assessment with selfreflection and peer feedback to optimize patient safety at all ages. The Canadian Anesthesiologists' Society (CAS) was among the first to implement national Guidelines to the Practice of Anesthesia ${ }^{10}$ to improve patient safety. The CAS adopted oximetry and capnography as routine intraoperative monitors for all patients without definitive evidence of effectiveness, yet their use by all anesthesiologists, independent of age, was intuitively the right thing to do. The current Guidelines ${ }^{10}$ specifically address "Administrative and Educational Policies" in several areas, such as continuing competence for nurses caring for patients having neuraxial anesthesia. It is unclear why these same Guidelines could not address minimum standards for the maintenance of competence for all anesthesiologists. If the problem is a failure of anesthesiologists to self-assess their ability to make decisions effectively in their work environment (at any age), then as a profession, we should build a meaningful system to assess competence.

\section{L'anesthésiologiste vieillissant: posons-nous la bonne question?}

Kahneman, ${ }^{1}$ un lauréat du prix Nobel, a reconnu que nous avions tous des avis personnels et des intuitions sur à peu près tout et que notre inclination naturelle nous pousse à croire que nous restons rarement totalement bouche bée. Quand une réponse satisfaisante à une question complexe n'est pas facilement disponible, une réponse intuitive humaine courante consiste à trouver une autre question apparentée à laquelle il est plus facile de répondre. Dans ce numéro du Journal, Baxter et coll. ${ }^{2}$ abordent un sujet important qui a trait au professionnalisme des anesthésiologistes et du déclin de leurs performances cliniques lié à l'âge. Les auteurs proposent une stratégie «proactive» que les départements hospitaliers et les groupes de professionnels devraient prendre en compte pour gérer ce problème. Ils identifient le fait que de nombreux anesthésiologistes souhaitent continuer à exercer au-delà de l'âge de 65 ans et que l'aptitude des médecins à s'autoévaluer s'est avérée inadéquate pour protéger la sécurité des patients. Nous devons nous demander si cette incapacité à s'autoévaluer est limitée à ce groupe d'âge (il y a d'abondantes données probantes montrant que cette lacune n'est pas dépendante de l'âge) et si on peut attendre de la stratégie proposée qu'elle améliore la sécurité globale des patients.

Le fondement de la synthèse entreprise par Baxter était partiellement en rapport avec les constatations de Tessler et coll. $^{3}$ pour qui les anesthésiologistes vieillissants (c'est-à-dire âgés de plus de 65 ans) avaient un risque 1,5 fois plus élevé (après ajustement pour l'exposition) d'être considérés responsables dans des contentieux, par rapport à leurs collègues plus jeunes. Il est important de noter que le degré des torts, définis par les dédommagements financiers associés aux cas litigieux, était plus élevé pour les patients 
des anesthésiologistes plus âgés. Tessler ${ }^{3}$ a reconnu que sa méthodologie (et donc ses conclusions) avait des limites dues à la faible fréquence des litiges en pratique clinique, à l'environnement de travail inconnu des différents groupes et à la méconnaissance du mécanisme sous-jacent à l'augmentation du risque. Il a spéculé que le risque augmenté chez les anesthésiologistes plus âgés pourrait avoir été lié à une plus grande fatigabilité, entraînant une baisse de la vigilance, des temps de réponse allongés, une formation continue moins intense ou de moins bonnes pratiques de communications. Dans un commentaire, $\mathrm{Eger}^{4}$ a remarqué qu'un autre facteur confondant pourrait avoir été que les deux groupes (c'est-à-dire les plus de 65 ans et les moins de 65 ans) étaient fondamentalement différents en termes de formation et d'ensembles de compétences depuis le début même de leur carrière professionnelle. Lorsque l'on traite de questions sociales et légales délicates, nous sommes tous d'accord pour dire que l'on ne doit pas tirer des conclusions possiblement erronées sur des individus à partir de l'analyse statistique de groupes.

Il y a, toutefois, d'autres données en faveur des conclusions de Tessler selon lesquelles l'âge des anesthésiologistes est un facteur contributif à la diminution des résultats favorables en pratique clinique. Campbell et coll., 5 par exemple ont rapporté que $52 \%$ des plaintes de patients enregistrées au Royaume-Uni par le General Medical Council impliquaient des anesthésiologistes qualifiés depuis plus de 20 ans alors que seulement $39 \%$ de la cohorte étaient âgés de plus de 45 ans. Baxter et coll. ${ }^{2}$ ont posé une question importante et néanmoins complexe qui requiert qu'on l'analyse sérieusement. Nous ne pouvons pas, et ne devrions pas, nier qu'il puisse y avoir un problème non reconnu ayant trait à certains anesthésiologistes, qui soit une baisse des habiletés cliniques liée à l'âge. Néanmoins, pour élaborer une démarche stratégique destinée à améliorer la sécurité des patients, il nous faut identifier la nature, l'étendue et les conséquences du problème ainsi que sa solution.

Les auteurs ${ }^{2}$ passent en revue les changements physiologiques survenant avec le vieillissement et spéculent que la cause de l'augmentation du risque pour la sécurité du patient pourrait être des limites de perceptions (par exemple, des déficits auditifs ou visuels) ou un déclin cognitif qui pourrait influencer le jugement et la prise de décision. Ils proposent que les «données probantes disponibles» suggèrent une «démarche proactive » qui pourrait conduire à un changement dans la « position par défaut qui consiste à continuer d'exercer jusqu'à ce qu'un problème soit reconnu ». Ils proposent que les départements et groupes de pratique concernés demandent à leurs collègues vieillissants de subir un bilan annuel, y compris une démonstration de compétence à l'aide de simulation, pour assurer la poursuite de leur travail dans le cadre hospitalier. Même si ce type de démarche proactive peut être intuitivement attrayante, les auteurs reconnaissent que l'on manque de données pour suggérer que seulement les anesthésiologistes les plus âgés ont ces types de déficits ou qu'une évaluation basée sur la simulation pourrait être un outil efficace de dépistage envers spécifiquement les anesthésiologistes les plus âgés.

Les auteurs suggèrent également que les anesthésiologistes vieillissants puissent continuer à pratiquer en raison d'un manque d'épargne pour leur retraite. Il est intéressant de noter que notre société professionnelle a identifié ce problème il y a déjà plusieurs décennies et que les membres anciens de la profession ont créé le CAMAF ou Canadian Anaesthetists' Mutual Accumulating Fund ${ }^{6}$ pour contribuer à fournir une sécurité financière. Si les considérations financières sont la cause sous-jacente de la poursuite de l'activité clinique des anesthésiologistes, de façon peut-être inappropriée, notre profession devrait de nouveau examiner les pensions ou de nouvelles stratégies de retraite.

Avant d'adopter la stratégie suggérée par Baxter et coll., ${ }^{2}$ nous devons demander si leur réponse est le reflet de la bonne question (c'est-à-dire une préoccupation pour la sécurité globale des patients) ou s'ils ignorent que leur réponse attire l'attention sur une question plus facile (c'est-à-dire la compétence des anesthésiologistes vieillissants travaillant dans les hôpitaux) puisque Kahneman ${ }^{1}$ a suggéré que cela était notre tendance naturelle. Il faut insister sur le fait que toutes les études montrent que le nombre absolu d'événements secondaires (attribués aux anesthésiologistes) qui aboutissent à des plaintes ou des contentieux est plus élevé pour les anesthésiologistes plus jeunes, bien que leur fréquence soit relativement plus importante chez les spécialistes âgés de plus de 65 ans.

La stratégie proactive ${ }^{2}$ suggérée est une intervention basée à l'hôpital qui peut aider à rassurer le public en montrant que la sécurité des patients est optimisée dans les hôpitaux. Cependant, une évolution prévisible est que les anesthésiologistes vieillissants qui souhaitent continuer à pratiquer puissent choisir de quitter les hôpitaux et de pratiquer dans des environnements isolés " hors hôpital ». Un exercice hors hôpital est souvent dépourvu de la sécurité que sont les interactions collégiales quotidiennes qui caractérisent la pratique de groupe et les conseils et soutiens concernant les décisions de soins aux patients. Cet environnement peut aussi limiter les occasions de développement professionnel continu. Je suggèrerais que l'environnement de pratique des anesthésiologistes de tous âges est un facteur important dans la genèse des évènements indésirables. Cela pourrait être une autre variable confondante de l'étude de Tessler, ${ }^{3}$ car des anesthésiologistes âgés peuvent avoir travaillé dans des 
environnements isolés à haut risque. Avant de proposer que la stratégie proposée améliore la sécurité globale des patients, nous devons considérer son possible impact sur la société. Cela pourrait protéger les hôpitaux (et les groupes de pratique) d'un certain risque médico-légal, mais la sécurité globale des patients par rapport à des complications de l'anesthésie pourrait ne pas être améliorée. Je suggèrerais que la sécurité anesthésique puisse être déterminée à tout âge par la compétence du fournisseur au sein de l'environnement dans lequel il ou elle exerce. Le problème d'un effectif vieillissant en anesthésiologie est un problème professionnel systémique qui nécessite une démarche professionnelle systémique.

Warner ${ }^{7}$ a également considéré que l'environnement dans lequel travaillent les anesthésiologistes vieillissants est un important facteur et a proposé une modification par étapes de la charge de travail pour réduire l'impact de la fatigue. Ce changement par étapes incluait une réduction des gardes de nuit ainsi qu'une limitation d'activité pour certains domaines et du nombre d'heures travaillées, ces deux mesures étant destinées à créer un environnement favorisant des soins optimaux aux patients. Où sont les données suggérant que les évènements indésirables, liés spécifiquement aux anesthésiologistes vieillissants, surviennent de façon disproportionnée la nuit ou après de longues gardes? Même si, comme cela a déjà été mentionné, cette idée parait intuitivement attrayante. Une autre caractéristique de l'environnement d'une salle d'opération moderne est la présence croissante d'assistants d'anesthésie agissant comme membres de l'équipe de soins anesthésiques et apportant un soutien important pour la gestion des problèmes cliniques difficiles. L'optimisation de l'environnement de la salle d'opération peut permettre à des anesthésiologistes de tous âges de fournir aux patients des soins plus sécuritaires sans avoir à «confisquer les clés ». ${ }^{7} \mathrm{Si}$ le problème fondamental est l'incapacité des anesthésiologistes à s'autoévaluer et à faire correspondre leurs compétences avec les besoins des soins des patients dans l'environnement dans lequel ils travaillent, c'est alors l'environnement et leurs capacités d'auto-évaluation qui sont les clés permettant d'améliorer la sécurité pour les anesthésiologistes de tous âges.

Kahneman ${ }^{1}$ a également analysé la prise de décision dans des disciplines telles que la médecine. Il a fait remarquer que l'acquisition d'une expertise dans des tâches complexes prend du temps «parce que l'expertise dans un domaine ne repose pas sur une seule habileté, mais plutôt sur une grande série de mini-habiletés ». Il a suggéré que le même professionnel peut être un grand expert de certaines tâches dans son domaine et rester novice dans d'autres. C'est là l'un des problèmes fondamentaux soulevés par l'utilisation d'évaluations basées sur de la simulation seule comme mesure de la compétence globale: elles ne peuvent pas évaluer l'éventail complet des «mini-habiletés » que doit posséder un anesthésiologiste praticien et elles n'ont jamais été validées pour cela. Warner ${ }^{7}$ soutient que de nombreux médecins plus âgés compensent bien leur manque de «connaissances mises à jour » grâce à leur expérience. Kahneman ${ }^{1}$ a conclu que la probabilité que des professionnels développent une expertise intuitive, qui caractérise une prise de décision clinique basée sur l'expérience, dépend de la qualité et de la vitesse du retour d'information dans le cadre clinique et aussi de la possibilité d'avoir une activité suffisante. Il a indiqué de façon spécifique que les anesthésiologistes bénéficiaient du retour d'information en pratique clinique parce que les effets de leurs actions avaient toutes les chances d'être immédiatement visibles. Selon lui, les anesthésiologistes sont mieux placés que de nombreux autres spécialistes pour développer des habiletés intuitives utiles, plus particulièrement du fait du retour d'information inhérent à leur travail. Cela suppose qu'ils puissent tirer parti de ces occasions de retour d'information ou pratiquer dans un environnement simulé. La simulation pour les anesthésiologistes de tous âges peut ne pas avoir uniquement de la valeur pour s'exercer à des habiletés techniques et non techniques ou à la gestion des crises cliniques rares et imprévisibles dans un environnement contrôlé. Un avantage largement méconnu de la simulation est de donner aux anesthésiologistes cliniciens la possibilité de réfléchir sur leur propre pratique et de recevoir un retour d'information sur leurs performances de la part de leurs collègues. Si la justification de l'adoption d'une stratégie « proactive » pour répondre aux problèmes posés par les anesthésiologistes vieillissants est leur manque de capacité à autoévaluer ces habiletés, alors c'est sans doute ce problème-là qu'il faut résoudre. Kahneman ${ }^{1}$ a insisté sur le fait que, dans de nombreux domaines de l'exercice du métier, les professionnels n'ont pas « appris à identifier les situations et les tâches dans lesquelles leur intuition pourrait les trahir » et ces limites non identifiées de l'habileté professionnelle permet en partie d'expliquer pourquoi des experts (de tous âges) sont souvent trop confiants dans leurs capacités.

Nous devons faire attention à ne pas nier qu'il existe des problèmes liés au vieillissement dans l'effectif des anesthésiologistes, mais nous devons comprendre et élaborer des stratégies pour répondre à la nature précise et à l'étendue du problème. Tessler ${ }^{3}$ avait raison: il faut poursuivre les recherches. D'une certaine façon, il est ironique qu'au Canada notre spécialité se dirige vers une évaluation des résidents basée sur les compétences ${ }^{8}$ alors que Baxter et coll. ${ }^{2}$ s'intéressent à un modèle basé sur l'âge (basé sur le temps) pour ce qui concerne le maintien de la compétence professionnelle. L'idée pourrait paraitre 
attrayante, mais est-ce la réponse à la bonne question pour ce qui concerne la sécurité des patients? Dans une situation semblable, la relation appropriée entre les heures de travail chirurgical des résidents et les évènements indésirables a été supposée de façon intuitive et les gardes ont été réduites au nom de la sécurité des patients. Néanmoins, des analyses récentes de cette expérience ont montré que l'amélioration attendue des résultats pour les patients ne s'est pas confirmée: en fait, les résultats pourraient être moins bons! ${ }^{9}$ Cela souligne simplement le fait que nous devons avoir conscience des conséquences inattendues de décisions basées sur des hypothèses que nous pensons vraies, mais qui sont fondées sur des notions préconçues inconnues.

Une autre option à envisager comme démarche « proactive » face au problème serait de s'assurer que tous les anesthésiologistes aient des évaluations régulières orientées sur les résultats de leurs patients tout au long de leur carrière, non pas comme moyen de conserver des privilèges hospitaliers, dans un but de responsabilité professionnelle et de ligne directrice pour l'exercice de la profession. Cela ne se limiterait pas à une seule évaluation des hauts risques basée sur une simulation à l'âge de 65 ans, mais se traduirait plutôt à tous les âges par une évaluation par les collègues, comportant une réflexion personnelle et un retour d'information par les pairs pour optimiser la sécurité des patients. La Société canadienne d'anesthésiologie (SCA) a été parmi les premières à mettre en œuvre au niveau national des Lignes directrices pour la pratique de l'anesthésiologie ${ }^{10}$ pour améliorer la sécurité des patients. La SCA a adopté l'oxymétrie et la capnographie comme mesures standard de surveillance peropératoire pour tous les patients sans preuve certaine de leur efficacité; néanmoins, leur utilisation par tous les anesthésiologistes (et quel que soit leur âge) était intuitivement la bonne chose à faire. Les Lignes directrices ${ }^{10}$ actuelles abordent de façon spécifique les «Politiques administratives et d'éducation » dans plusieurs domaines, tels que le maintien de la compétence des infirmiers prenant soin de patients ayant une anesthésie neuraxiale. On ne voit pas pourquoi les mêmes Lignes directrices ne pourraient pas aborder des normes minimums de compétence pour tous les anesthésiologistes. Si le problème est que les anesthésiologistes ne parviennent pas à auto-évaluer leur capacité à prendre efficacement des décisions dans leur environnement de travail (quel que soit leur âge), alors, en tant que profession, nous devons bâtir un système adéquat pour évaluer les compétences.

Acknowledgements The author thanks Dr. David Mazer and Dr. David McKnight for their thoughtful comments on the manuscript. As a disclosure of potential bias and conflict of interest, the author is compelled to acknowledge that, although he may perceive that he is mid-career, his colleagues continue to remind him that he is a "senior member of the Department".

Conflicts of interest None declared.

Conflits d'intérêts Aucun déclaré.

\section{References}

1. Kahneman D. Expert intuition: when can we trust it. In: Kahneman D. Thinking, Fast and Slow. Anchor Canada; 2013.

2. Baxter AD, Boet $S$, Reid D, Skidmore $G$. The ageing anesthesiologist: a narrative review and suggested strategies. Can J Anesth 2014: 61: this issue. DOI: 10.1007/s12630-0140194-x.

3. Tessler MJ, Shrier I, Steele RJ. Association between anesthesiologist age and litigation. Anesthesiology 2012; 116 : 574-9.

4. Eger EI 2nd. Maybe it isn't ageing. Anesthesiology 2012; 117 : 1138-9.

5. Campbell G, Rollin AM, Smith AF. Cases relating to anaesthetists handled by the UK General Medical Council in 2009: methodological approach and patterns of referral. Anaesthesia 2013; 68: 453-60.

6. Anonymous. Canadian Anaesthetists' Mutual Accumulating Fund. Can Med Soc J 1958; 78: 800.

7. Warner MA. More than just taking the keys away. Anesthesiology 2012; 116: 501-3.

8. McKenna $J$, Rosen D. Competency-based professionalism in anesthesiology: continuing professional development. Can J Anesth 2012; 59: 889-908.

9. Ahmed N, Devitt KS, Keshet I, et al. A systematic review of the effects of resident duty hour restrictions in surgery: impact on resident wellness, training, and patient outcomes. Ann Surg 2014; 259: 1041-53.

10. Merchant $R$, Chartrand D, Dain S, et al. Guidelines to the practice of anesthesia - revised edition 2014. Can J Anesth 2014; 61: 46-71. 